\title{
Paradoxical response during a Para-Hisian pacing in a case with fasciculo-ventricular pathway
}

\author{
Debabrata Bera ${ }^{1}$, Calambur Narasimhan ${ }^{2}$, Ayan Kar $^{1}$, and Joyanta Ghosh ${ }^{1}$ \\ ${ }^{1}$ RTIICS \\ ${ }^{2}$ Asian Institute of Gastroenterology
}

November 19, 2021

Title: Paradoxical response during a Para-Hisian pacing in a case with fasciculo-ventricular pathway.

Short title : Para-Hisian pacing in fasciculo-ventricular pathway

Authors:

Debabrata Bera, DM, Dept of Cardiology, RTIICS, Kolkata. India.

Calambur Narasimhan, DM, AB, Dept of Cardiology, AIG Hospitals, Hyderabad, India.

Ayan Kar, DNB, Dept of Cardiology, RTIICS, Kolkata. India.

Joyanta Ghosh, DM, Dept of Cardiology, RTIICS, Kolkata. India.

\section{Corresponding author:}

Debabrata Bera. Rabindranath Tagore International Institute of Cardiac Sciences (RTIICS), 124 Mukundapur, Kolkata. West Bengal, India. PIN- 700099.

Ph: +91-8013894181, Fax: +91-33 2426 4204.Email- debu2000pgi@gmail.com

Key words: Fasciculo-ventricular accessory pathway; Para-Hisian Pacing; Paradoxical response.

Word count: 909.

Funding: None

Conflict of interest: None

Consent has been taken from the patient.

Data availability statement:

All raw data and recording during the case are available for review.

Case

A 28-year-old man presented with orthodromic atrioventricular reentrant tachycardia and underwent successful ablation of manifest right posterolateral atrioventricular accessory pathway (AP) [Appendix 1]. After successful ablation, the HV was still found to be short (26 ms) and a fasciculo-ventricular pathway (FVP) was suspected. The HV interval was short and fixed and the QRS morphology remained unchanged during decremental atrial pacing (Fig 1). He also had junctional beats of identical QRS morphology (Appendix 2) confirming the diagnosis of FVP. There was an underlying right bundle branch block (RBBB) in sinus rhythm resurfacing after the ablation (Fig 1). 
Para-Hisian pacing (PHP) was performed to confirm abolition of retrograde AP conduction. We observed the following responses reproducibly during a PHP. Pacing at distal His bundle (HB) region was performed @ $450 \mathrm{~ms}$ of pacing cycle length (PCL) with decreasing pacing output starting from $10 \mathrm{~mA} @ 2 \mathrm{~ms}$ pulse width [Fig 2]. What is the mechanism behind the paradoxical response of Stimulus to A-EGM (SA) prolongation?

\section{Commentary:}

The usual responses in any FVP and the atypical findings in this case are discussed below:

Due to loss of insulation in FVP, the fascicular system is often captured irrespective of high or low output [1]. Therefore, isolated myocardial capture (Vc) is difficult to achieve from the HB region. In absence of isolated myocardial capture PHP becomes uninterpretable. However, in this case we could repeatedly demonstrate Vc and infer on the route of VA conduction [Discussed in next paragraph].

The PHP was performed from distal HB region (HISd bipole) [Fig 3]. In the absence of an atrioventricular AP, the response during PHP in FVP should be nodal if the AV node has an intact VA conduction. This is due to the lack of retrograde conduction property of FVP $[2,3]$. In our case, the SA paradoxically prolonged with the narrower beat. The wider beats ( $1^{\text {st }}$ and $2^{\text {nd }}$ beats, QRS width of $130 \mathrm{~ms}$ ) appear to be resulting from myocardial capture $(\mathrm{Vc})$ and the narrower $3^{\text {rd }}$ beat $(110 \mathrm{~ms})$ from $\mathrm{H}+\mathrm{V}$ capture $(\mathrm{H}+\mathrm{Vc})$. Occasionally, the interpretation can be erroneous if the narrower beat actually results from a pure His capture (Hc) and the wider one due to $\mathrm{H}+\mathrm{Vc} / \mathrm{Vc}$. Hence, a careful analysis of QRS morphology is important. An isoelectric interval and narrow QRS morphology are useful clues for a pure His capture. Additionally, a dedicated catheter (other than the pacing catheter) at the HB region can be useful in this differentiation by showing the capture and release of His/V electrogram. Here, it was not available. However, there was no isoelectric interval in the narrower $3^{\text {rd }}$ beat excluding a pure Hc. In fact, later on we could demonstrate a pure His capture (Hc) which also corrected the underlying RBBB (analogous to a permanent His bundle pacing) [first beat in Fig 3, QRS width was $86 \mathrm{~ms}$ ]. Unfortunately, no interpretation about the VA conduction was made here as HRA catheter was the only atrial catheter at that point (CS catheter came out) and a proper assessment of atrial sequence could not be made.

We wondered about the mechanism of paradoxical SA prolongation in the narrower last beat in Fig 2. One of the causes of such response is nodal conduction during pure Hc [4,5]. In this case, pure Hc was excluded, as discussed above. The most likely mechanism for the paradoxical response here is due to decrement in the AV node which happened because the narrower $\mathrm{H}+\mathrm{Vc}$ beat followed a wider Vc beat. This, in turn, led to a shortening of $\mathrm{HH}$ interval and a decrement in the nodal VA conduction. When $\mathrm{H}+\mathrm{Vc}$ beat follows a $\mathrm{Vc}$ beat, it can lead to 20-40 ms shortening of HH interval due to earlier activation of the His bundle. This is the reason why it is always better to analyze PHP response in beats where a narrower $\mathrm{H}+\mathrm{Vc}$ complex is followed by a wider $\mathrm{Vc}$ beat and not the vice versa. Our proposition is mechanistically similar to the pitfall no. 6 described by Sheldon et al [5]. The other explanation of SA prolongation could be a development of retrograde RBBB. However, a $98 \mathrm{~ms}$ increment cannot be explained solely by a retrograde-RBBB and the $\mathrm{HH}$ shortening remains as the most probable mechanism. Another dedicated catheter with His electrogram, if available, could have rendered useful insights.

Interestingly, this phenomenon of SA prolongation due to slight $\mathrm{HH}$ shortening is rarely reported despite a common occurrence of narrower beat $(\mathrm{H}+\mathrm{Vc})$ following a wider beat $(\mathrm{V})$ during routine PHP. It is because this generally occurs in patients with sluggish VA conduction as in our case (VA Wenckebach was $430 \mathrm{~ms}$ ). The 20-40 ms shortening of HH interval made the effective pacing cycle length (PCL) for the last beat as 410-430 ms. The narrow gap between the PCL and VA Wenckebach interval led to the such finding. A briskly conducting node (VA) would not have so much impact with a slight HH shortening. The substantial prolongation might also occur when conduction shifts from fast to slow pathway [5,6], but the 'A-EGM' was still earliest at HISp bipole suggestive of decrement in fast pathway. Moreover, during a ventricular extrastimuli we did not observe any VA jump.

To complete the case, no tachycardia was inducible on baseline and isoprenaline infusion and no further ablation was attempted. In essence, this case highlights the importance of careful analysis of QRS morphology 
and HH intervals before interpreting a PHP response. This also illustrates that capture of different tissues in all combinations (like $\mathrm{Hc}, \mathrm{H}+\mathrm{Vc}, \mathrm{Vc}$ ) are possible even in presence of FVP. To the best of our knowledge, this is the first report of a paradoxical para-hisian response in a case with FVP which occurred due to inadvertent $\mathrm{HH}$ shortening.

\section{Figure legends:}

Fig 1: Short HV (26 ms) after ablation of the manifest atrioventricular AP. The small signal in front of the ventricular electrogram in His catheter was validated as ' $\mathrm{H}$ ' during decremental atrial pacing in the last beat. The fixed HV on surface ECG confirmed a FVP.

Fig 2: Para-Hisian pacing showing the first 2 beats were $\mathrm{Vc}$ and last one was $\mathrm{H}+\mathrm{Vc}$. There is a paradoxical SA prolongation in the narrower last beat.

Fig 3: Another protocol of PHP revealed a pure Hc in the first beat. The CS catheter was out, hence no assessment of VA was made.

\section{Supplementary file:}

Appendix 1: Baseline 12 lead ECG in sinus rhythm and atrial pacing before ablation

Appendix 1: A post ablation junctional beat showing the same QRS morphology suggestive of an infra-nodal pathway (likely FVP).

\section{References}

1. Hojo R, Fukamizu S, Sakurada H. Accurate evaluation of para-Hisian pacing in a patient with fasciculoventricular bypass. Europace. 2014 Oct;16(10):1475. doi: 10.1093/europace/euu203. Epub 2014 Aug 20. PMID: 25142740. 2. Asvestas D, Bazoukis G, Mililis P, et al. Fasciculoventricular bypass tracts: Electrocardiographic and electrophysiologic features.J Arrhythm. 2020;36(3):537-541. Published 2020 May 8. doi:10.1002/joa3.12355 3. Saito A, Kaneko Y, Nakajima T, et al. Electrocardiographic Characteristics of Fasciculoventricular Pathways: Analysis of Five Cases. Journal of Arrhythmia. 2010 . P 181-188.https://doi.org/10.1016/S1880-4276(10)80005-5. 4. Ho KL, Nair K, Chauhan VS. Paradoxical response during para-Hisian pacing. What is the mechanism? Heart Rhythm. 2012 Oct;9(10):1732-3. 5. Sheldon SH, Li HK, Asirvatham SJ, McLeod CJ. Parahisian pacing: technique, utility, and pitfalls. J Interv Card Electrophysiol. 2014 Aug;40(2):105-16. doi: 10.1007/s10840-014-9908-9. Epub 2014 Jun 11. PMID:

25011420. 6. Nair KKM, Namboodiri N, Paidi S, Balaguru S, Valaparambil A. Paradoxical increase of stimulus to atrium interval during para-Hisian pacing. What is the mechanism? J Cardiovasc Electrophysiol. 2020 May;31(5):1226-1228. 

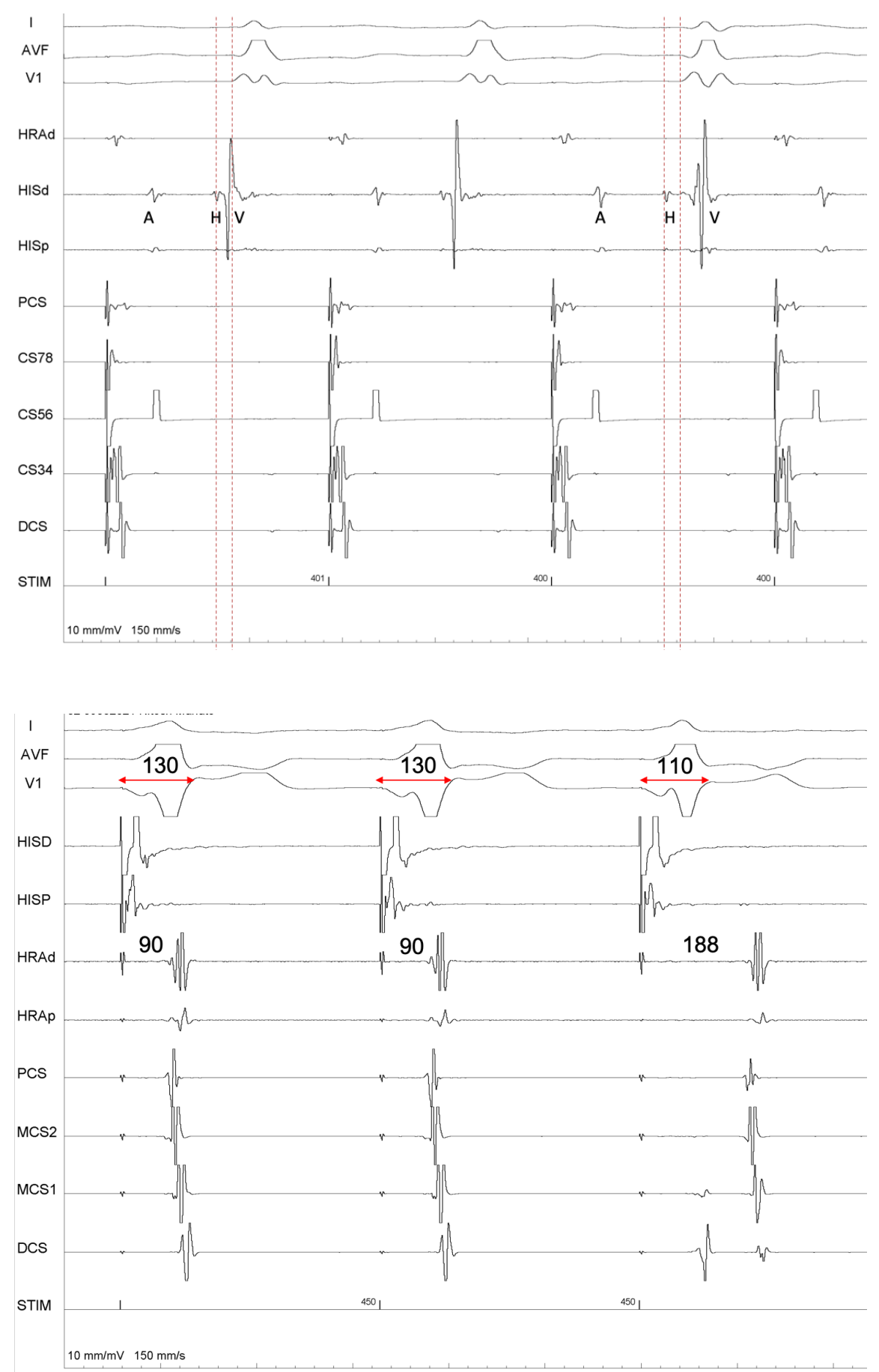


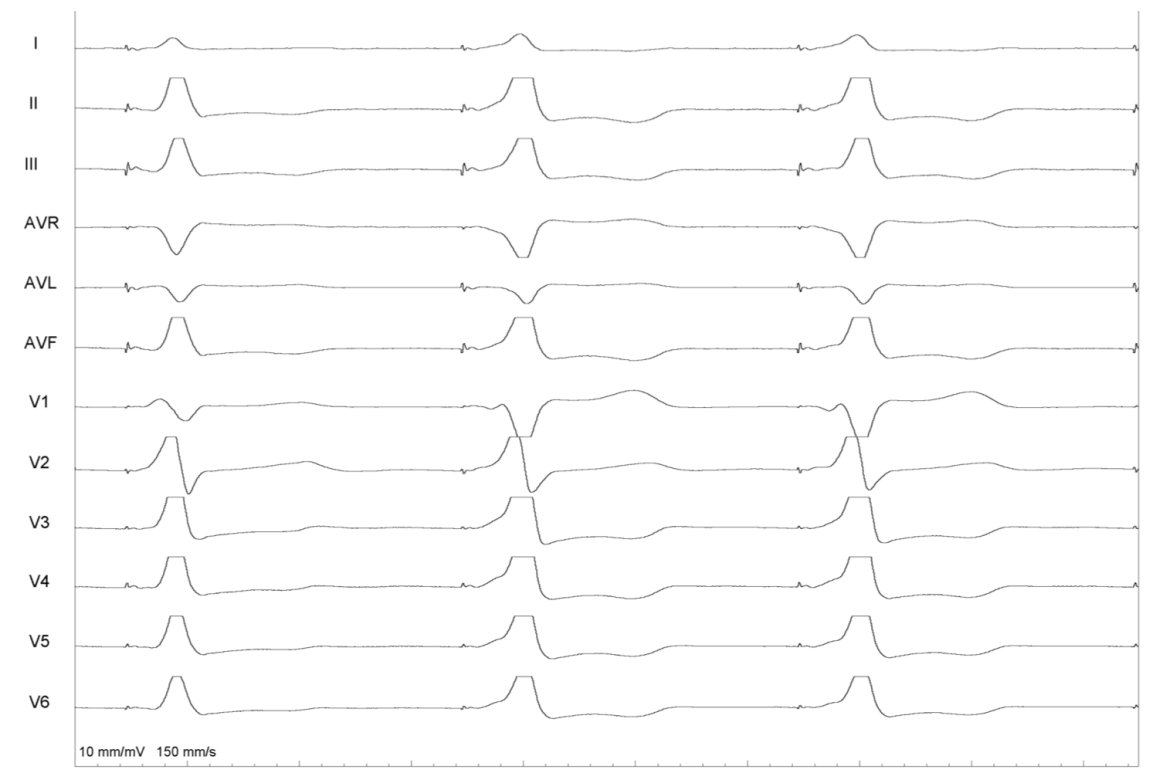

